\title{
Hospital information systems: A survey of the current situation in Iran
}

\author{
Mohsen Pourali ${ }^{1, ~ *, ~ A b b a s ~ G h o d r a t ~ P a n a h ~}{ }^{2}$ \\ ${ }^{1}$ Financial department, Mehr Hospital, Mashhad, Iran \\ ${ }^{2}$ Accounting group, Attar University, Mashhad, Iran
}

\section{Email address:}

Mohsen.poorali@gmail.com (M. Pourali),Sg_abbasgh@yahoo.com (A. G. Panah)

\section{To cite this article:}

Mohsen Pourali, Abbas Ghodrat Panah. Hospital Information Systems: A Survey of the Current Situation in Iran. International Journal of Intelligent Information Systems. Special Issue: Research and Practices in Information Systems and Technologies in Developing Countries. Vol. 3, No. 6-1, 2014, pp. 115-119. doi: 10.11648/j.jiiis.s.2014030601.31

\begin{abstract}
The main objective of hospital information systems (HIS) is to support hospital activities at practical, tactical and strategic levels. Computer based hospital information systems use computer hardware and software to collect, store, process, review and establish link with administrative data related to all hospital activities and to satisfy all the needs of clients. Considering the assessment and poll conducted in 2010 as well as the collected comments of physicians, hospital personnel, managers, researchers and computer engineering experts, HIS software in Iran consist of 10 organizational components and 10 service providing components with respect to domestic conditions. Study of HIS software providing companies in Iran during 2010 and their comparison with current status (2013) suggested that the number of HIS software manufacturing companies has experienced a 35\% growth and the number of outstanding and desirable HIS sub-systems in Iran shows a $142 \%$ growth while the percentage of acceptable and rejected sub-systems has decreased by $18 \%$ in spite of the $35 \%$ growth in the number of companies.
\end{abstract}

Keywords: Computer Based Hospital Information Systems, Organizational Components, Service Providing Components, Evaluation of HIS

\section{Introduction}

Hospital information system also known as HIS is referred to as health information system which is an integrated information system for management, storage, and retrieval of patients' information.

The objective of these systems is to provide support for hospital activities at practical, tactical and strategic levels. In computer based HIS, computer software and hardware are used to collect, store, process, review and establish link with administrative data in all hospital activities and to satisfy the needs of clients. The fact is that nowadays a hospital may not still continue to carry out the paperwork procedure of outpatients or inpatients in its traditional style, therefore establishment of HIS seems a necessity in order to increase accuracy and expedite the provision of services to patients, paperwork and circulation of documents in the hospitals and rapid file retrieval for various purposes such as research and studies of relevant students while facilitating access to medical documents. HIS resolves all the aforesaid problems associated with various medical wards in a hospital thus transforming the hospital from a traditional organization into a modern medical center.

In fact, the HIS is not merely restricted to medical issues. Hospitals are certainly formed of clinical and para-clinical divisions that have together with the support unit constituted an integrated unit that seeks a specified objective. Support units that are serially linked to the medical wards are all in line with provision of services to the patients. Support systems in hospitals are called Management Information Systems (MIS). Since support and treatment cycles supplement one another to enlarge the service provision cycle in the hospitals, a Hospital Information Management System (HIMS) has also been established which manages the entire medical and support cycles as well as all the activities in the hospitals.

Application of hospital information system in Iran was first introduced in 1999 as it was decided to initiate it for the 
very first time as a pilot project in the hospitals of Shahroud, Mashhad, Yazd and Zanjan. Having made this decision, the executive procedures of HIS project commenced in 1999 at Imam Hossein Hospital of Shahroud through conducting of preliminary studies and review of existing domestic and overseas experiences. The hardware platform was equipped during the first phase of the project in 2000 while admission and discharge stages were launched in the second phase and then, the mutual link between inpatient wards of para-clinical departments, patient invoice and identification code, completion of patient discharge stages, insurance and physicians fee schedule, and the clinical information of patients were covered by the hospital electronic system until the fifth phase in September 2001. As a result, the first electronic hospital of Iran was operationalized as a national pilot project in 2000 at the Imam Hossein 313-bed hospital of Shahroud [1].

In 2003 and following a call for Iranian experts working in countries of United Arab Emirates and Canada, Astan Ghods Razavi was first to apply the first HIMS software with trade name "Green Hospital Information System" at the newly founded 320-bed Razavi Hospital of Mashhad which included a perfect hospital information management system that provides integrated coverage of the entire medical and support cycles and activities while all the parts of the aforesaid software were installed and initiated in 2005 .

\section{Structure and Facilities of HIS Software in Iran}

While each hospital is considered an independent organization for provision of healthcare services, it is also influenced by domestic, regional and national healthcare regulations. Accordingly, hospital information systems are software systems that are highly influenced by domestic and local rules both in terms of design and assessment. Hence, it is observed that some software are manufactured in some place in the world and universally applied in other countries but this has not been the case with hospital information systems and may not be. Thus, assessment and evaluation of hospital information systems must be carried out in accordance with domestic standards either for procurement, ranking or design.

Considering the assessment and poll conducted by the Bureau of Statistics and Information Technology of the Ministry of Healthcare and Medical Education in 2010 through compilation of comments made by physicians, hospital staff, managers, researchers and computer engineering experts, the HIS software in Iran should include the following organizational components and service providing elements [4]:

\subsection{Organizational Components}

The organizational components consist of:

\subsubsection{Admission Information Sub-System}

This system is responsible for electronic documentation and admission of the patient as the first sub-system that provides services to the clients of the medical unit.

\subsubsection{Outpatient Information Sub-System}

While maintaining its integrity with HIS software of inpatient wards, this system must record and preserve the outpatient clients and information files.

\subsubsection{Hospital Ward Information Sub-System}

This sub-system must be able to cover the capabilities required for intensive care units and also be capable of establishing links with human resources, electronic medical record and electronic nursing record sub-systems.

\subsubsection{Pharmacy Information Sub-System}

This sub-system must be capable of recording the inpatient and outpatient information of medicines in addition to management of medicine storehouses.

\subsubsection{Laboratories Information Sub-System}

This sub-system is equipped with recording and provision of tests results for inpatient and outpatient while maintaining an integrated link with electronic medical record.

\subsubsection{Radiology Information System}

In addition to recording and providing radiology test results of inpatients and outpatient, this sub-system must be directly linked to the picture archiving communication systems (PACS).

\subsubsection{Operation Room Information System}

This sub-system must be capable of managing the operation room with regards to admission of patients and also be able to record the required data during the surgery.

\subsubsection{Medical Document Information System}

Preparation of precise and perfect information and statistics on status, quantity and quality of the services provided at the clinics, para-clinics and medical wards to be used by the state organizations engaged in the area of healthcare, managers and decision makers of hospitals are among the capabilities of this Sub-System.

\subsubsection{Discharge Information System}

Discharge Information System calculates the fee of medical services provided in the medical wards.

\subsubsection{Hospital Nutrition Sub-System}

It is used to mechanize the link between functions of nutrition department with prescriptions of the treating physician related to nutrition. In some HIS software, the aforesaid sub-system manages the meals of staff, patient escorts and those of other clients in addition to management of meals for patients.

The link between organization components is presented in Figure 1 [5]. 


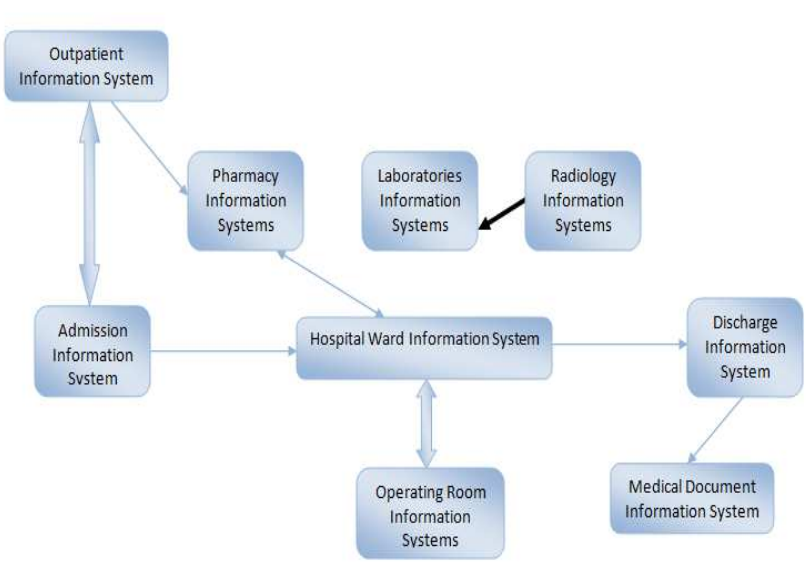

Figure 1. The link between organizational components

\subsection{Service Providing Components}

The service providing components consist of:

\subsubsection{Electronic Medical Record}

The electronic medical record is one of the hospital requirements the data from which are used by the entire hospital wards including medical wards, clinics and paraclinics. Although the medical information of each individual are recorded at different wards of the hospital, integrity of information in the medical record increases the efficient operation and usefulness of medical data while these information may be used in case of following referrals of patient. Some government authorities in Iran such as the Social Security Organization and some of HIS manufacturers such as Farasoo Company have already paved the way for submission of information of electronic medical record to the patient for use in other medical centers while maintaining confidentiality.

\subsubsection{Electronic Nursing Record}

This one deals with recording and archiving of information from patient's clinical (nursing) reports and is used similar to the medical electronic record in order to provide the data required for diagnosis and treatment. Physicians and nurses are the main beneficiaries of this system. The difficulties of using electronic nursing records in Iran include the statutory problems and the necessity for manuscript reports which has led to failure in use and entry of precise and perfect data of nurses in the HIS software due to double entry and timeconsumption.

\subsubsection{Bed Management Information System}

Management of hospital beds calls for integrated and efficient relation between medical, admission and discharge units which is a facility provided by this sub-system.

\subsubsection{Personnel and Scheduling Information System}

Personal, employment, professional position and rank of personnel may be recorded via this sub-system. It also manages the schedules of medical and support personnel and is effectively linked to wage and salary system.

\subsubsection{Decision Making Support Sub-System}

One of the most efficient applications of using HIS software is its contribution to decision making. Diagnosis and treatment decision-support systems are currently being applied in HIS software of Iran.

\subsubsection{Terminology Service}

Supporting the coding systems for consistency with international organizations and unification of individuals' understanding of medical diagnoses and procedures is one of the most significant components in hospital information systems. Clinical diagnoses coding (ICD10), Clinical Procedures Coding (CPT) and Medical Laboratory Observations Coding (LOINC) are currently used in majority of HIS software of Iran.

\subsubsection{Communication Service}

The communication service establishes an infrastructure for transfer of data between organizational and service providing components at inter-hospital communication level in case the databanks of these two components are separated so that safe and secure transfer of data may become possible. Standard data transfer protocols such as HL7 are also underlined in the area of healthcare in case of external transfers which is also provided in some of the HIS software of Iran.

\subsubsection{Telemedicine Service}

Telemedicine is the application of information and telecommunications technology in order to provide remote medical services. Web-based systems have facilitated this external feature while most HIS software in Iran are equipped with this inter-hospital facility.

\subsubsection{Resource Management Information Sub-system}

Inventory of goods and healthcare commodities is one of the most fundamental resources of hospitals as the necessity for control of inputs and outputs, consumption, deterioration or expiration of goods is only decided in storehouse systems. In case of some HIS software that has incorporated the integrated data of support systems, other inventory of goods and commodities may also be controlled which prevents repetitive data entry and re-recording of data.

\subsubsection{Security Service}

Observance of information security and access limit of users are essential objectives taking into account the integrity if information in HIS software. The link between organization components is presented in Figure 2 [5].

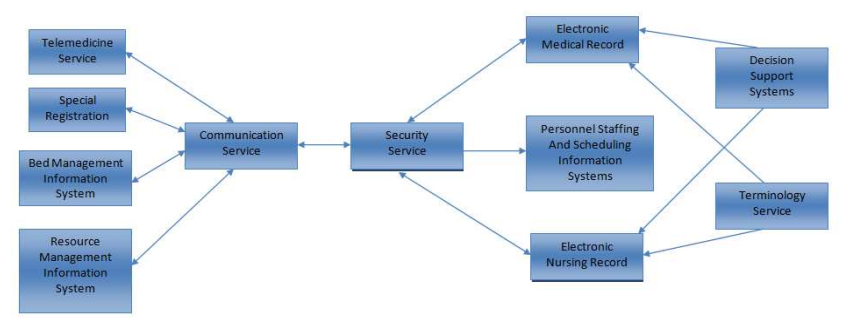

Figure 2. The link between service providing components 
The link between organization components and service providing components is presented in Figure 3 [5].

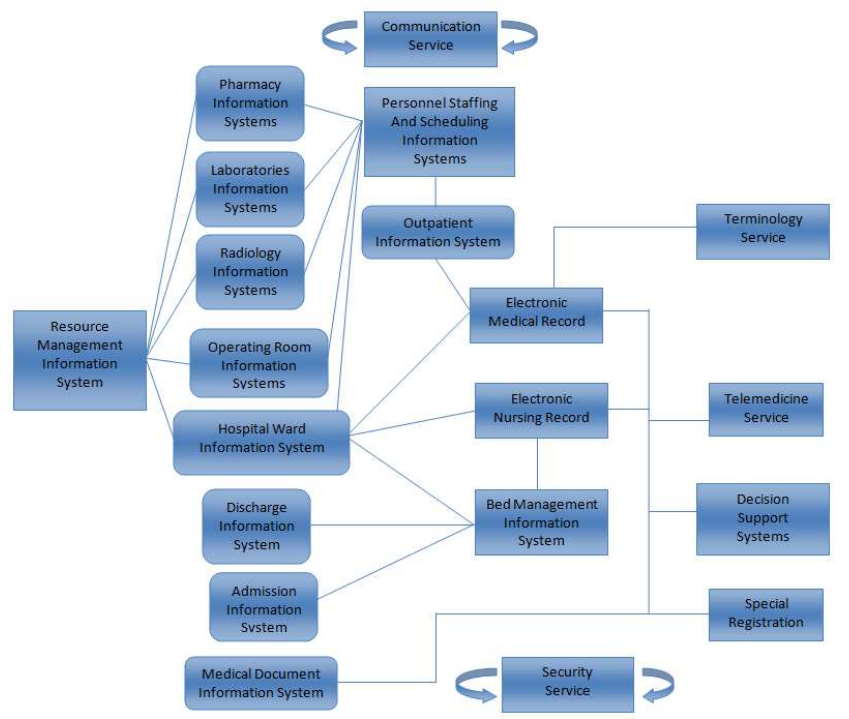

\section{Current Status of HIS software in Iran}

According to the study conducted by the Bureau of Statistics and Information Technology of Ministry of Healthcare and Medical Education in 2010 [3], the status of HIS software in Iran comprises 20 HIS software manufacturing companies (Table1).

Scores one, two and three have respectively been allocated to favorite, acceptable and rejected statuses. As the conducted assessment reveals, electronic medical records and discharge with 1.3 and 1.4 averages are the most favorite sub-systems while telemedicine and communication service are the most inadequate sub-systems with averages of 2.5 and 2.7, respectively.

Based on the assessment, an average of $6.7 \%$ of companies falls into favorite category while $5.9 \%$ and $7.5 \%$ of companies are categorized as acceptable and rejected, respectively.

Figure 3. The link between organizational and service providing components

Table 1. List of 20 HIS software and their relevant scores

\begin{tabular}{|c|c|c|c|c|c|c|}
\hline Components & Rejected & Acceptable & Favorite & Variance & Average & Sum \\
\hline Electronic Medical Record & 2 & 17 & 1 & 0.2 & 2.1 & 41 \\
\hline Electronic Nursing Record & 7 & 9 & 4 & 0.6 & 2.2 & 43 \\
\hline Personnel Staffing and Scheduling Information System & 12 & 4 & 4 & 0.7 & 2.4 & 48 \\
\hline Terminology Service & 7 & 0 & 13 & 1 & 1.7 & 34 \\
\hline Security Service & 1 & 15 & 4 & 0.2 & 1.9 & 37 \\
\hline Communication Service & 17 & 0 & 3 & 0.5 & 2.7 & 54 \\
\hline Resource Management Information System & 6 & 3 & 11 & 0.8 & 1.8 & 35 \\
\hline Bed Management Information System & 7 & 7 & 6 & 0.7 & 2.1 & 41 \\
\hline Admission Information Sub-System & 6 & 6 & 8 & 0.7 & 1.9 & 38 \\
\hline Outpatient Information Sub-system & 10 & 1 & 9 & 1 & 2.1 & 41 \\
\hline Hospital Ward Information System & 8 & 6 & 6 & 0.7 & 2.1 & 42 \\
\hline Pharmacy Information System & 5 & 6 & 9 & 0.7 & 1.8 & 36 \\
\hline Laboratories Information System & 6 & 6 & 8 & 0.7 & 1.9 & 38 \\
\hline Radiology Information System & 9 & 9 & 2 & 0.5 & 2.4 & 47 \\
\hline Medical Document Information System & 3 & 0 & 17 & 0.5 & 1.3 & 26 \\
\hline Discharge Information System & 3 & 2 & 15 & 0.6 & 1.4 & 28 \\
\hline Hospital Nutrition System & 10 & 4 & 6 & 0.8 & 2.2 & 44 \\
\hline Average & 7.5 & 5.9 & 6.7 & & & \\
\hline
\end{tabular}

The most recent status of HIS software in Iran according to the study conducted by the Bureau of Statistics and Information Technology of Ministry of Healthcare and
Medical Education in 2013 [2], the status of HIS software in Iran comprises 27 HIS software manufacturing companies (Table2).

Table 2. List of 27 HIS software and their relevant scores

\begin{tabular}{|c|c|c|c|c|c|c|}
\hline Components & Rejected & Acceptable & Favorite & Outstanding & Variance & Average \\
\hline Electronic Medical Record & 6 & 4 & 10 & 7 & 1.23 & 2.33 \\
\hline Electronic Nursing Record & 9 & 5 & 5 & 8 & 1.56 & 2.56 \\
\hline Personnel Staffing and Scheduling Information System & 14 & 2 & 3 & 8 & 1.85 & 2.81 \\
\hline Terminology Service & 1 & 9 & 11 & 6 & 0.70 & 2.19 \\
\hline Security Service & 0 & 4 & 3 & 20 & 0.56 & 1.41 \\
\hline Communication Service & 10 & 2 & 6 & 9 & 1.72 & 2.48 \\
\hline Resource Management Information System & 6 & 2 & 5 & 14 & 1.54 & 2.00 \\
\hline
\end{tabular}




\begin{tabular}{|c|c|c|c|c|c|c|}
\hline Components & Rejected & Acceptable & Favorite & Outstanding & Variance & Average \\
\hline Bed Management Information System & 4 & 1 & 12 & 10 & 1.04 & 1.96 \\
\hline Admission Information Sub-System & 2 & 5 & 6 & 14 & 1.00 & 1.81 \\
\hline Outpatient Information Sub-system & 5 & 6 & 2 & 14 & 1.53 & 2.07 \\
\hline Hospital Ward Information System & 7 & 7 & 4 & 9 & 1.49 & 2.44 \\
\hline Pharmacy Information System & 4 & 6 & 8 & 9 & 1.16 & 2.19 \\
\hline Laboratories Information System & 2 & 5 & 9 & 11 & 0.92 & 1.93 \\
\hline Radiology Information System & 6 & 7 & 7 & 7 & 1.26 & 2.44 \\
\hline Medical Document Information System & 1 & 5 & 4 & 17 & 0.86 & 1.63 \\
\hline Discharge Information System & 3 & 5 & 7 & 12 & 1.11 & 1.96 \\
\hline Hospital Nutrition System & 8 & 0 & 7 & 12 & 1.67 & 2.15 \\
\hline Treatment Accounting Information System & 5 & 5 & 10 & 7 & 1.14 & 2.30 \\
\hline Average & 6.05 & 4.81 & 6.10 & 10.05 & & \\
\hline
\end{tabular}

Scores one, two and three have respectively been allocated to favorite, acceptable and rejected statuses. As the conducted assessment reveals, security and medical records systems with 1.63 and 1.4 averages are the most favorite sub-systems while telemedicine and decision-support are the most inadequate sub-systems with averages of 3.37 and 2.85, respectively.

Based on the assessment, an average of $10.5 \%$ of companies falls into excellent category while $6.10 \%, 4.81 \%$ and $6.05 \%$ of companies are categorized as favorite, acceptable and rejected, respectively.

Comparatively speaking and considering the following table, the numbers of HIS software manufacturing companies and favorite and excellent HIS software have grown by $35 \%$ and $142 \%$ from 2010 to 2013 , respectively (Table 3 ).

Table 3. Overview of number of HIS software and their status in 2010 and 2013

\begin{tabular}{lcccc}
\hline & Rejected & Acceptable & Outstanding and Desirable & No. of Companies \\
\hline 2010 Assessment & 7.45 & 5.9 & 6.65 & 20 \\
2013 Assessment & 6.05 & 4.81 & 16.14 & 27 \\
Deviation & $(1.40)$ & $(1.09)$ & 9.49 & 7 \\
Deviation Percentage & $\%(18.82)$ & $\%(18.48)$ & $\% 142.75$ & $\% 35$ \\
\hline
\end{tabular}

\section{Discussion and Conclusion}

In this article we reviewed the current status of Hospital Information Systems in Iran. Our study included the comparison of characteristics of about 20 information systems. Based on the assessment, an average of $10.5 \%$ of companies falls into excellent category while $6.10 \%, 4.81 \%$ and $6.05 \%$ of companies are categorized as favorite, acceptable and rejected, respectively. Considering the assessment and poll conducted in 2010 as well as the collected comments of physicians, hospital personnel, managers, researchers and computer engineering experts, HIS software in Iran consist of 10 organizational components and 10 service providing components with respect to domestic conditions. Study of HIS software providing companies in Iran during 2010 and their comparison with current status (2013) suggested that the number of HIS software manufacturing companies has experienced a $35 \%$ growth and the number of outstanding and desirable HIS subsystems in Iran shows a $142 \%$ growth while the percentage of acceptable and rejected sub-systems has decreased by $18 \%$ in spite of the $35 \%$ growth in the number of companies.

\section{References}

[1] D., Zeydanloo. Hospital Information System. The Center for Research, Development and Policy Making of Faculty of Medical Sciences of Shahid Beheshti University, 2012.

[2] H., Riazi, S., Abedian, A., Bitaraf. Performance Assessment of Hospital Information Systems. The Bureau of Statistics and Information Technology of the Ministry of Healthcare and Medical Education, 2013.

[3] Anonymous, Results of Performance Analysis of Hospital Information System Software. The Bureau of Statistics and Information Technology of the Ministry of Healthcare and Medical Education, 2010.

[4] Anonymous, Framework of Performance Assessment of Hospital Information Systems. The Bureau of Statistics and Information Technology of the Ministry of Healthcare and Medical Education, 2010.

[5] Christoph Schlachter. New Business Models for E-Healthcare and the role of trust. Master thesis submitted to the Department of Informatics University of Zurich, Zurich, Switzerland, 2004. 